\title{
Effect of Abuse History on Pain Reports and Brain Responses to Aversive Visceral Stimulation: An fMRI Study
}

\author{
YEHUDA RINGEL, ${ }^{*}$ DOUGLAS A. DROSSMAN, ${ }^{*}$ JANE L. LESERMAN, ${ }^{*, \neq}$ BRANDALL Y. SUYENOBU, ${ }^{\S}$ KATHY WILBER," \\ WEILI LIN," WILLIAM E. WHITEHEAD, * BRUCE D. NALIBOFF, ${ }^{\S}$ STEVEN BERMAN, ${ }^{\S}$ and EMERAN A. MAYER ${ }^{\S}$ \\ *UNC Center for Functional Gl and Motility Disorders and ־Department of Psychiatry, University of North Carolina, Chapel Hill, North Carolina; §UCLA Center for \\ Neurovisceral Sciences and Women's Health, Departments of Medicine, Physiology, and Psychiatry, David Geffen School of Medicine at UCLA, Los Angeles, \\ California; and "Division of Neuroradiology, University of North Carolina, Chapel Hill, North Carolina
}

Background \& Aims: Abuse history is common in irritable bowel syndrome (IBS) and is associated with greater pain reporting, psychologic distress, and poorer health outcome. These effects may be mediated by enhanced responses to aversive visceral stimuli. We investigated the effects of IBS and abuse history on pain reporting and brain activation in response to rectal distentions. Methods: Ten female patients with IBS and 10 controls were included. Half of patients in each group reported a history of abuse. Brain functional magnetic resonance imaging (fMRI) images and pain ratings were obtained during rectal distentions. Statistical parametric mapping identified activation in subregions of the dorsal cingulate cortex and covariation with rated pain. Results: (1) Distention-elicited pain correlated with anxiety and activation of the posterior (PCC) and middle (MCC) dorsal cingulate subregions. (2) Subjects with a history of abuse showed greater activation in the left MCC $(P=$ $.022 ; \mathrm{t}=5.61)$ and PCC $(P=.033 ; \mathrm{t}=5.00)$ than subjects without abuse. (3) Those with IBS and abuse reported greater pain than all others $(P=.004)$, had more activity in the left MCC $(P=.021 ; t=5.29)$ and PCC $(P=.049 ; t=4.81)$, and had less activity in the left supragenual anterior cingulate (sACC) $(P=.01 ; \mathrm{t}=$ 4.86). Conclusions: Pain ratings during rectal distention are associated with activation of dorsal cingulate regions implicated in homeostatic afferent processing, and prior abuse enhances this activation. $\mathrm{Pa}$ tients with IBS and abuse report more pain, greater MCC/PCC activation, and reduced activity of a region implicated in pain inhibition and arousal (sACC). These findings suggest a possible explanation for the clinical observation of greater pain reporting and poorer outcome in IBS patients with a history of abuse.

A ccording to the biopsychosocial model, the clinical presentation and outcome of irritable bowel syndrome (IBS) are shaped by the interaction of biologic and psychosocial factors. ${ }^{1}$ With regard to the latter, several studies have shown a high prevalence of abuse history among female patients in gastrointestinal (GI) clinics ${ }^{2-4}$ and particularly in patients with IBS and other functional GI disorders. ${ }^{3-7}$ Sexual and/or physical abuse is reported in up to $50 \%$ of patients with IBS and other functional GI disorders seen in referral GI clinics, which is a higher prevalence than is seen in the general population or in patients with organic GI disorders. ${ }^{6,7}$ Furthermore, we have reported that more severe abuse (eg, rape, life threat) is significantly more frequent in IBS than in organic disorders. ${ }^{3,4}$ Predictive analyses show that psychosocial and behavioral features play a prominent role in determining the severity of painful functional bowel disorders, including IBS, ${ }^{8,9}$ and a history of abuse is associated with greater pain reporting, greater psychologic distress, poorer health status, and poorer outcome regardless of the GI diagnosis. ${ }^{3,10}$ One possible reason for greater pain reporting and poorer health outcome might relate to increased perceptual responses to rectosigmoid stimuli in patients with abuse history.

The relatively recent application of functional brain imaging to study brain-gut interactions and IBS pathophysiology has made it possible to characterize the role of the central nervous system (CNS) in mediating the effects of cognitive and emotional factors on perception of visceral signals, including visceral pain. ${ }^{11,12}$ Published preclinical and clinical evidence suggests that the CNS can modulate the behavioral responses to and the perception of visceral pain and that this central pain modulation may adversely affect the clinical presentation of IBS. ${ }^{11-14}$ An example is a case report from our group of a young woman with abuse history and IBS who showed increased activation of the anterior cingulate gyrus that was clinically associated with high pain scores and psychosocial distress, all of which improved after successful treatment. ${ }^{15}$ Even though different experimental paradigms have yielded differences in the patterns of altered brain

Abbreviations used in this paper: fMRI, functional magnetic resonance imaging; MCC, midcingulate cortex; PCC, posterior cingulated cortex; SACC, supragenual anterior cingulate cortex.

(C) $\mathbf{2 0 0 8}$ by the AGA Institute 0016-5085/08/\$34.00 doi:10.1053/j.gastro.2007.11.011 
responses to visceral stimulation in IBS patients, enhanced activation of limbic and paralimbic regions, including the dorsal cingulate cortex, have been reported by several investigators in different samples (reviewed in Mayer et $\mathrm{al}^{14}$ ). With the high prevalence of abuse in IBS patients and the evidence that abuse history has a synergistic effect on producing greater pain scores and other adverse health outcomes in IBS, ${ }^{3}$ it is plausible that an abuse history may be associated with enhanced activation of the dorsal cingulate cortex and brain networks that have been implicated in central pain amplification in IBS and/or diminished activity in regions such as the supragenual anterior cingulate cortex (sACC) and associated networks that play a role in the inhibition of pain and emotional arousal. ${ }^{11-14}$ Consistent with this hypothesis is the extensive literature on the compromised ability of subjects with a trauma history to regulate brain circuits involved in emotional arousal. ${ }^{16}$

Differential activation of subregions of the cingulate gyrus by a wide variety of experimental stimuli, including somatic and visceral pain and emotional stimuli, has been reported. ${ }^{14,16-22}$ The dorsal cingulate cortex includes 3 major subregions: the most posterior region (posterior cingulate cortex, $\mathrm{PCC}$ ), the middle third of the cingulate cortex (midcingulate cortex, MCC), and the most rostral region, located just above the genu of the corpus callosum (sACC). These subregions are illustrated in Figures 1-4. Activation of the middle region (MCC), frequently accompanied by deactivation of the posterior cingulate (PCC) and peri- and infragenual cingulate cortices, has been consistently reported in the somatic pain literature. Therefore, MCC together with the insular cortex and the thalamus have been referred to as part of the "central pain matrix." 23 As pointed out by Craig et al, because not only pain but a wide range of painful and nonpainful, interoceptive, and exteroceptive stimuli (all of which inform the brain about the homeostatic state of the organism) are processed within the same network, ${ }^{24}$ a more appropriate terminology may therefore be the "homeostatic afferent processing network." 14 In contrast, the most rostral aspect of the dorsal cingulate (sACC) and the adjacent medial prefrontal cortex have been implicated in negative feedback regulation of the amygdala and arousal. ${ }^{25}$ The same brain regions, which are rich in $\mu$ opioid receptors, have also been implicated in the regulation of endogenous pain inhibition circuits. ${ }^{26}$ Thus, sACC/medial prefrontal cortex play important roles both in the regulation of emotional arousal and of pain perception. ${ }^{27} \mathrm{~A}$ more detailed discussion of the major functions of the subregions of the cingulate gyrus is available in Paus. ${ }^{22}$

In the current study, we investigated the effects of IBS diagnosis and a history of abuse on pain reporting and regional brain activation in response to nonpainful and painful rectal distentions. We hypothesized that abuse history is associated with greater pain reports and with alterations in activation of dorsal cingulate gyrus subregions during aversive visceral stimulation. This hypothesis-driven study focuses on a limited number of preplanned group comparisons (especially the IBS with abuse against all others) and on 3 specific predetermined subregions of the cingulate gyrus (ie, sACC, MCC, PCC).

We specifically wanted to test the following 3 related hypotheses: (1) IBS patients with a history of abuse (compared with those without such a history) will show synergistic effects of the IBS diagnosis and abuse history on the increased activation of the MCC, a brain region typically associated with the emotional and motivational aspects of pain responses and with increased arousal ${ }^{14}$; (2) subjects with abuse, in particular IBS subjects with abuse, will show a reduced activation of the sACC/medial prefrontal cortex region, similar to reported findings in certain subjects with trauma history'16; and (3) that the increased activation of the MCC will be positively correlated with subjective pain ratings.

\section{Materials and Methods}

We studied 20 right-handed female subjects aged $18-65$ (mean age, $27.6 \pm 10.0$ years) years. All subjects were recruited by advertising at UNC campus and UNC hospital general and GI clinics. Ten subjects had a diagnosis of IBS, and 10 subjects were without IBS. Non-IBS subjects served as controls and were completely asymptomatic with regard to any GI symptoms. Half of the subjects in each group reported a history of sexual and/or physical abuse (sexual abuse and physical abuse, $\mathrm{n}=8$; sexual abuse only, $\mathrm{n}=1$; physical abuse only, $\mathrm{n}=1$ ). Abused subjects were selected for a history of severe abuse (8 of 9 raped, 5 of 9 serious life threats), and most had multiple sex and/or physical abusive events. IBS subjects (all subtypes) met ROME II criteria and had active symptoms at least over the past 2 weeks.

Assessment of lifetime sexual and physical abuse history was done using a standard interview administered by a trained investigator (J.L.). This measure is based on our previous studies ${ }^{10,28,29}$ and has been shown to correlate with many indicators of poor health. ${ }^{10,29,30}$ Sexual abuse was defined as rape (penetration) or unwanted touching of genitalia where force or threat of harm was present, and physical abuse was defined as a life-threatening attack.

Psychological distress was measured using the Global Symptom Index (GSI) of the SCL-90 asking about distress during the past month. ${ }^{31}$ We used the raw score from the summary measure of all 90 items ranging from 0 (low distress) to 4 (high distress) and the anxiety and depression subscales. ${ }^{31}$

\section{Rectal Distention Protocol}

The rectal distention protocol consisted of 3 sets of repeated 39 seconds rectal distentions separated by a 39-second rest period in the following order: 4 nonpain- 
ful $(15 \mathrm{~mm} \mathrm{Hg})$ distention trials, 4 painful $(50 \mathrm{~mm} \mathrm{Hg})$ distention trials, and 5 alternating nonpainful/painful $(15,50,15,50,15 \mathrm{~mm} \mathrm{Hg})$ distention trials. At the end of each set of distentions, subjects were asked to rate their pain experience during the distention trial on a 6-point rating scale: 0 , none; 1 , weak; 2 , mild; 3 , moderate; 4 , strong; 5 , intense. Selection of these distention pressures was based on our previous studies showing that $50-\mathrm{mm}$ $\mathrm{Hg}$ distentions are associated with pain sensation and that $15-\mathrm{mm} \mathrm{Hg}$ distentions are noticeable but not associated with painful sensation in subjects with IBS. ${ }^{32}$ This is also consistent with numerous reports from other groups. $^{33}$

\section{Brain Image Acquisition}

All images were acquired on a Siemens 1.5 Tesla MR scanner (Siemens Medical Systems, Munich, Germany) with a gradient strength of $25 \mathrm{mT} / \mathrm{m}$. A T2* weighted echo planar imaging sequence was used to acquire images. The imaging parameters were as follows: repetition time, 3 seconds; echo time, 54 milliseconds; slice thickness, $5 \mathrm{~mm}$; field of view, $192 \times 192 \mathrm{~mm}^{2}$, with a matrix size of $64 \times 64$; and 16 slices. A block design was used with 39 seconds for the resting (OFF) and rectal balloon distention $(\mathrm{ON})$ states. Statistical parametric mapping (SPM2) (Wellcome Trust Centre for the Study of Cognitive Neurology ${ }^{34,35}$ ) was used to preprocess the images and to identify significant effects.

\section{Image Preprocessing}

Functional magnetic resonance images (fMRI) were processed prior to analysis by realignment, normalizing, and smoothing procedures. The realignment procedure corrected for head movement by aligning the images for each subject with the central image of the first block (image 50 of block 1). All images were then reoriented to approximate a match to the origin and orientation of the Montreal Neurological Institute (MNI) EPI template image. Graphic representations of the realignment were assessed to identify excessive movement $(>3$ $\mathrm{mm}$ translation and/or $>3^{\circ}$ rotation) within any block. Images were removed from further analysis if these limits were exceeded. Visual inspection of the realigned images revealed excessive truncation of the field of view in one subject with IBS but no history of abuse, so this subject was dropped from brain image analyses. Realigned images from the remaining 19 subjects were moved into a standardized brain image space developed at the Montreal Neurological Institute (MNI-space) using linear and nonlinear transformations. Normalized images were spatially smoothed with a 5-mm, full-width, half maximum kernel.

\section{Data Analysis}

We compared patients' self-reported pain ( 0 , no pain, to 5 , intense pain) for the 3 blocks of distentions: 1 , nonpainful (15 mm Hg); 2, painful $(50 \mathrm{~mm} \mathrm{Hg})$; and 3, alternating $(15 \mathrm{~mm} \mathrm{Hg}$ alternating with $50 \mathrm{~mm} \mathrm{Hg})$ using paired $t$ tests. Because the brain imaging analysis of the response to painful distention used data from both the consistently painful (4 events at $50 \mathrm{~mm} \mathrm{Hg}$ ) and the 2 painful $50-\mathrm{mm} \mathrm{Hg}$ distention events from the alternating painful/nonpainful trial, we used the average of patients' pain ratings for the painful distentions and alternating distentions blocks.

Comparisons between study subgroups (IBS $[\mathrm{n}=10]$ vs no IBS $[\mathrm{n}=10]$, abuse $[\mathrm{n}=10]$ vs no abuse $[\mathrm{n}=10]$, and IBS and abuse $[\mathrm{n}=5]$ vs all others $[\mathrm{n}=15])$ regarding self-reported pain during rectal distentions (average of consistently painful and alternating painful) were done using $t$ tests. Because age, education, and race were not related to pain reports $(P>.09)$, we did not control for these variables. Functional data from each subject were initially analyzed by entering all images into a fixed-effects analysis, specifying the timing sequence and intensity of each inflation. Brain responses to each level of inflation, as compared with the baseline period, were modeled at each voxel by convolving the timing sequence of those inflations with a synthetic hemodynamic response function (http://www.fil.ion.ucl.ac.uk/ $\mathrm{spm})$. Linear contrasts were applied to the parameter estimates for each condition with the resulting $t$ scores at each voxel constituting the statistical parametric map for that condition.

Contrast images representing effects of the $15-\mathrm{mm} \mathrm{Hg}$ and $50-\mathrm{mm} \mathrm{Hg}$ inflations were generated for each subject and entered into second level, random effects analyses. Because this allows between subject variance to be quantified, results can be generalized to populations from which subjects were randomly sampled. Second-level analyses used 2-sample $t$ tests to compare blood-oxygenlevel dependent (BOLD) responses between experimental groups and correlation analyses to assess covariation of responses with reported pain. Peak voxel $t$ values were interrogated in each of 3 a prior regions of interest (ROIs) within the dorsal cingulate gyrus of each cerebral hemisphere. These were defined by the middle (MCC) and posterior (PCC) thirds of the cingulate cortex, and the portion of the most anterior cingulate cortex above the genu of the corpus collosum (supragenual; sACC). Anterior cingulate cortex below the genu could not be assessed because of inferior midline inhomogeneity artifacts that extended almost to the genu in the fMRI images of several subjects. All ROIs were drawn by author S.B. on the MNI 152-brain-average T1 template image using the MEDx (Sensor Systems, Sterling, VA) medical imaging program. Peak $t$ scores associated with $P$ values $<.05$ after false discovery rate (FDR) correction for multiple comparisons within the region of interest (ROI-FDR $P)$ constituted evidence for significant effects. Effects with $P<.1$ are reported as suggestive trends. Exploratory whole-brain analyses evaluated brain activity $(P<.05$ 
Abuse vs nonAbuse

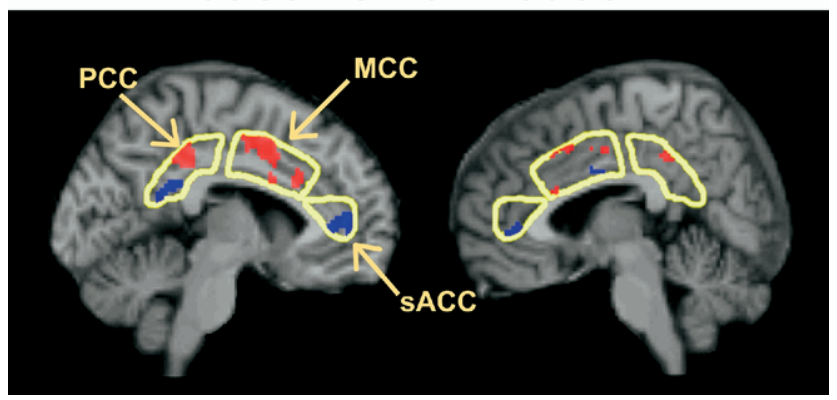

Figure 1. Response to painful $(50 \mathrm{~mm} \mathrm{Hg})$ distentions. Greater activation in left MCC (ROI-FDR, $P=.022, t=5.61$, at $-8,6,42)$ and left PCC (ROI-FDR, $P=.033, t=5.00$, at $-12,-34,42)$ in subjects with a history of abuse compared with subjects without abuse history. The lower activation in the left SACC was not statistically significant after volume correction. Note that the 3 regions presented (SACC, MCC, and PCC) together form the dorsal cingulate cortex.

uncorrected) outside the 3 predetermined regions of interest. In addition, the whole-brain corrected probability associated with the spatial extent of the cluster (set of contiguous voxels) that included each significant or suggestive ROI peak was assessed to ensure the effect did not represent a highly localized process.

\section{Results}

The average age of our female study sample was $27.6 \pm 10.0$ years, average education was $13.8+2.0$ years, and $65 \%$ were of white ethnicity. Abused subjects were less educated than the nonabused subjects (12.8 vs 14.7 school years, respectively) $(P=.03)$, and those with IBS and abuse had less education than all other subjects (12.0 vs 14.3 school years, respectively) $(P=.02)$. Subjects' age was not different between the study subgroups.

\section{Behavioral Measures}

Table 1 shows the effects of IBS and abuse on pain reporting following painful and nonpainful distentions

IBS vs nonIBS

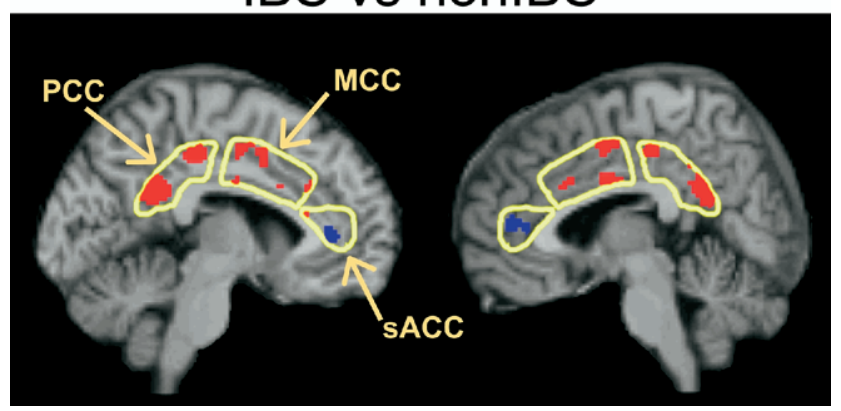

Figure 2. Response to painful $(50 \mathrm{~mm} \mathrm{Hg})$ distentions. Trends for greater left (ROI-FDR, $P=.061, \mathrm{t}=4.42$, at $-8,-46,24)$ and right PCC activation (ROI-FDR, $P=.092, t=4.13$, at $8,-44,28$ ) in the IBS group compared with the non-IBS group. There were no significant effects in the MCC or SACC.
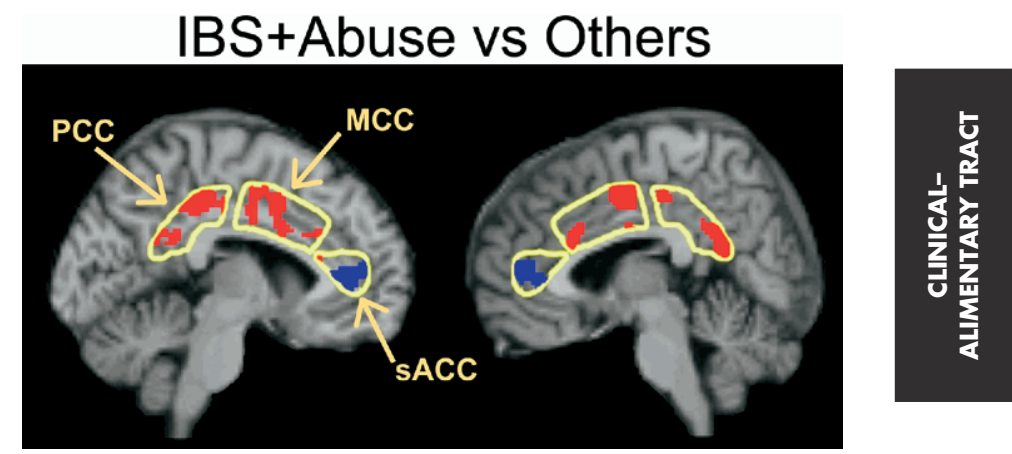

Figure 3. Response to painful $(50 \mathrm{~mm} \mathrm{Hg})$ distentions. Greater activation in left MCC (ROI-FDR, $P=.021, t=5.29$, at $-6,6,42)$ and left PCC (ROI-FDR, $P=.049, t=4.81$, at $-12,-34$, and 42 ), less activation in the left sACC (ROI-FDR, $P=.01, \mathrm{t}=4.86$, at $-6,42,6)$ and right sACC (ROI-FDR, $P=.078, \mathrm{t}=3.13$, at $8,42,8)$, and a trend for greater activation in right PCC (ROI-FDR, $P=.077, \mathrm{t}=3.82$, at $2,-52,20)$ in subjects with both IBS and a history of abuse compared with other subjects. Note that the SACC effects that can be seen in Figures 1 and 2 as trends attain significance when comparing those with both IBS and abuse against those with only one or neither condition.

as well as anxiety and depression scores during the previous month (SCL-90R). All subjects reported significantly more pain with the painful distentions (average of the $50-\mathrm{mm} \mathrm{Hg}$ painful distention block and the alternating $15 / 50-\mathrm{mm} \mathrm{Hg}$ block) than with the nonpainful (15 $\mathrm{mm} \mathrm{Hg})$ distentions $(3.64 \pm 0.83$ vs $1.80 \pm 0.62, P<$ .0001 , respectively). There were no significant differences in pain reports following the repeated $50-\mathrm{mm} \mathrm{Hg}(3.75 \pm$ 0.85 ) and the alternating $15 / 50-\mathrm{mm} \mathrm{Hg}$ distention blocks $(3.53 \pm 0.98)$; therefore, Table 1 shows the average of pain reports in response to the 2 blocks.

Relationship of pain reports with IBS diagnosis and history of abuse. When comparing IBS with no IBS or abuse to no abuse, there were no significant differences in pain reporting during the painful and nonpainful distentions. However, patients with the combination of IBS diagnosis and a history of abuse reported significantly more pain during the painful distentions than all

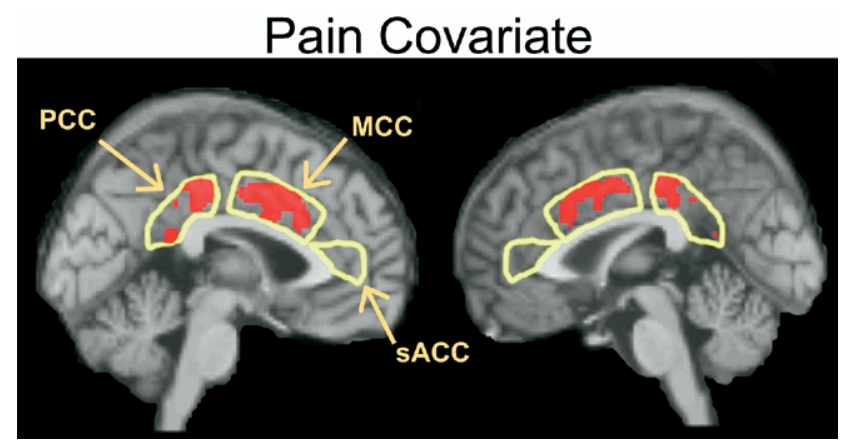

Figure 4. A positive correlation between all subjects' pain reports during $50-\mathrm{mm} \mathrm{Hg}$ rectal distention and activation of MCC and adjacent PCC. The correlation was significant in the left MCC (ROI-FDR, $P=$ $.048, t=4.60$, at $-12,-4,38$ ). There was no relationship between pain reports and activity in the SACC region. 
Table 1. Reported Pain Following Rectal Distentions and Psychologic Distress by IBS and Abuse

\begin{tabular}{|c|c|c|c|c|}
\hline & \multicolumn{2}{|r|}{ Pain ratings ( $0-5$ range) following } & & \\
\hline & \multirow{2}{*}{$\begin{array}{l}\text { Nonpainful distentions } \\
\quad(15 \mathrm{~mm} \mathrm{Hg})\end{array}$} & \multirow{2}{*}{$\begin{array}{l}\text { Painful distentions (average distention events during } \\
\text { painful and alternating blocks) }\end{array}$} & \multicolumn{2}{|c|}{ Psychologic distress (SCL-90R) } \\
\hline & & & Anxiety & Depression \\
\hline & Mean (SD) & Mean (SD) & Mean (SD) & Mean (SD) \\
\hline All subjects $(\mathrm{N}=20)$ & $1.80( \pm 0.62)^{a}$ & $3.64( \pm 0.83)$ & $0.73( \pm 1.05)$ & $0.96( \pm 1.03)$ \\
\hline IBS $(n=10)$ & $2.00( \pm 0.67)$ & $3.93( \pm 0.66)$ & $1.06( \pm 1.19)$ & $1.29( \pm 1.28)$ \\
\hline No IBS $(n=10)$ & $1.60( \pm 0.52)$ & $3.35( \pm 0.91)$ & $0.40( \pm 0.80)$ & $0.62( \pm 0.59)$ \\
\hline Abuse $(n=10)$ & $1.90( \pm 0.74)$ & $3.80( \pm 1.05)$ & $1.07( \pm 1.30)$ & $1.39( \pm 1.22)^{b}$ \\
\hline No abuse $(n=10)$ & $1.70( \pm 0.48)$ & $3.48( \pm 0.53)$ & $0.38( \pm 0.60)$ & $0.53( \pm 0.59)$ \\
\hline IBS/abuse $(n=5)$ & $2.20( \pm 0.84)^{c}$ & $4.50( \pm 0.31)^{d}$ & $1.42( \pm 1.53)$ & $1.82( \pm 1.57)^{c}$ \\
\hline All others $(n=15)$ & $1.67( \pm 0.49)$ & $3.35( \pm 0.74)$ & $0.50( \pm 0.76)$ & $0.67( \pm 0.62)$ \\
\hline
\end{tabular}

${ }^{a} P<.0001$, nonpainful vs painful distentions during painful and alternating blocks.

${ }^{b} P=.067$, abuse different from nonabuse.

${ }^{c} P=.094$, IBS/abuse different from all others on nonpainful distentions and depression.

${ }^{d} P=.004$, IBS/abuse different from all others on painful distentions.

other groups (eg, abuse/no IBS, no abuse/no IBS, and IBS/no abuse) ( 4.5 vs $3.35, P=.004)$. There was also a trend for women with IBS and abuse to have more pain during the nonpainful trial compared with all other women ( 2.20 vs 1.67 , respectively, $P=.094)$. Note that patients with abuse tended to have more depression during the past month than patients without abuse (SCL90R-depression, 1.39 vs 0.53 , respectively, $P=.067$ ).

Relationship of psychologic distress with pain reports. In the group as a whole, pain ratings after nonpainful distentions were strongly correlated with anxiety $(r=0.69, P=.0007)$, depression $(r=0.70, P=.0007)$, and global distress $(r=0.68, P=.0009)$. Pain reported after the painful distentions (average of consistent and alternating pain) showed moderate and statistically significant correlations with the subjects' general level of anxiety $(r=0.48, P=.03)$. There were trends for reported pain after painful distentions to be correlated with global psychologic distress (GSI) $(r=0.44, P=.055)$ and depressive symptoms $(r=0.42, P=.068)$.

\section{Brain Activation}

Analysis of brain activation by diagnosis of IBS and history of abuse. Group differences were assessed for each level of rectal distention comparing the brain response between 10 abuse vs 9 no-abuse subjects, 9 IBS vs 10 non-IBS subjects, and the 5 subjects with both IBS and abuse vs the remaining 14 subjects. There were no significant group differences in response to the repeated nonpainful $(15 \mathrm{~mm} \mathrm{Hg})$ distention.

Abuse vs nonabuse. During painful $(50 \mathrm{~mm} \mathrm{Hg})$ distention, subjects with a history of abuse (including IBS with abuse and controls with abuse) had greater activation in the left MCC (ROI-FDR, $P=.022, \mathrm{t}=5.61$, at $-8,6,42)$ and the left PCC (ROI-FDR, $P=.033$, $\mathrm{t}=$ 5.00 , at $-12,-34,42)$. Both voxels were part of a wholebrain cluster of 990 voxels $(P=.027)$ that included $11 \%$ of the left MCC, $11 \%$ of the right MCC, $12 \%$ of the left
PCC, and 6\% of the right PCC but no evidence for significant effects in sACC (Figure 1).

IBS vs non-IBS. There were no significant group differences in any of the ROIs. However, there were trends for greater PCC activation during painful $(50 \mathrm{~mm}$ $\mathrm{Hg}$ ) distention in the IBS group in both the left (ROIFDR, $P=.061, \mathrm{t}=4.42$, at $-8,-46,24)$ and the right hemisphere (ROI-FDR, $P=.092, \mathrm{t}=4.13$, at $8,-44,28$ ). Both voxels were part of a whole-brain cluster of 6078 voxels $(P<.0005)$ that included $23 \%$ of the left PCC and $29 \%$ of the right PCC but no evidence for effects in neighboring MCC (4\% left MCC, $5 \%$ right MCC) or in sACC (Figure 2).

IBS with abuse vs all others. During painful ( 50 $\mathrm{mm} \mathrm{Hg}$ ) distention, subjects with both IBS and a history of abuse showed greater activation than the remaining subjects in the left MCC (ROI-FDR, $P=.021, \mathrm{t}=5.29$, at $-6,6,42)$ and the left PCC (ROI-FDR, $P=.049, \mathrm{t}=4.81$, at $-12,-34,42$ ), with a trend in the right PCC (ROIFDR, $P=.077, \mathrm{t}=3.82$, at $2,-52,20)$. All 3 peak voxels were part of a whole-brain cluster of 6665 voxels $(P<$ .0005 ) that included $20 \%$ of the left MCC, $10 \%$ of the right MCC, $30 \%$ of the left PCC, and $37 \%$ of the right PCC. In the sACC, IBS patients with a history of abuse showed less activation (left sACC, ROI-FDR, $P=.01, \mathrm{t}=$ 4.86, at $-6,42,6$ ) (right sACC ROI-FDR, $P=.078$, $\mathrm{t}=$ 3.13 , at $8,42,8)$. Both voxels were part of a whole-brain cluster of 2599 voxels $(P<.0005)$ that included $45 \%$ of the left sACC and $45 \%$ of the right sACC (Figure 3 ).

\section{Relationship of Brain Activation With Rectal Distention and Pain Reports}

Across all 19 subjects, there was a positive correlation between subjects' pain reports during $50-\mathrm{mm} \mathrm{Hg}$ rectal distention and activation of the 2 cingulate subregions, MCC and adjacent PCC. A significant correlation was found in the left MCC (ROI-FDR, $P=.048, \mathrm{t}=4.60$, $r=0.75$, at $-12,-4,38)$. This voxel was part of a 
whole-brain cluster of 4934 voxels (spatial extent, $P<$ .0005 ) that included $51 \%$ of the left MCC, $27 \%$ of the right MCC, $27 \%$ of the left PCC, and $29 \%$ of the right PCC. There was no evidence of any relationship between pain reports and activity in the more rostral cingulate region, the sACC (Figure 4).

\section{Discussion}

A history of abuse and other psychosocial factors is common in patients with IBS and can exacerbate GI symptoms, influence illness experience, and affect treatment outcome. ${ }^{2-9,36,37}$ The association of a history of abuse with the diagnosis of IBS and its poorer outcome has suggested to some that abuse history may be associated with central mechanisms of pain amplification, resulting in greater pain reports and greater clinical behavioral responses to painful visceral stimuli.6,32,38 Here, we expand our previous observations on the effects of a history of abuse on GI symptoms, illness experience, and treatment outcome $e^{2-9,36,37}$ as well as on its effect on brain responses. ${ }^{15,32,38}$

First, we demonstrated that patients with IBS and a history of abuse report significantly more pain in response to aversive rectal distentions compared with patients with IBS or abuse history alone. Our findings are consistent with clinical and research observations that patients with an abuse history have greater pain reports and poorer health status regardless of a functional or organic diagnosis. ${ }^{3}$

Second, we were able to demonstrate that aversive rectal distention among patients with abuse history + IBS has stronger effects on the dorsal cingulate gyrus than for those with IBS diagnosis or a history of abuse alone (Figure 3). This was also the group associated with higher pain reporting compared with controls or those solely with IBS or abuse. These findings may help explain our previous outcome data showing that abuse history is additive to the adverse effects of a functional or organic disorder on health status (eg, greater pain, psychologic distress, quality of life, and health care utilization) over those with either condition alone. ${ }^{3}$ The synergistic activation effects for these patients of enhanced MCC and adjacent PCC activation may reflect greater CNS responses to perturbations of internal or external homeostasis (reviewed in Mayer et $\mathrm{al}^{14}$ ). The brain receives information about the homeostatic state of the body from a large number of spinal and vagal afferents, and this information is processed within the homeostatic afferent processing network. ${ }^{14}$ Any actual or anticipated perturbation of homeostasis is associated with activation of the central network including the dorsal cingulate gyrus. MCC activation is associated with increased arousal, affective stimulus ratings, and increased motivational drive.

Such a conceptualization of dorsal cingulate is consistent with studies showing that this brain region is activated during social threats and unpleasant and threaten- ing noxious stimulation, including visceral and somatic pain, ${ }^{14,18-21}$ as well as other biologic threats to homeostasis such as cold air, hunger, ${ }^{39}$ and hypoglycemia. ${ }^{40}$ Even though the dorsal cingulate receives ascending projections from the thalamus and from the insula, activity in this region is also modulated by influences from the amygdala and ascending noradrenergic projections from the locus coeruleus complex. ${ }^{22}$ The latter influence can regulate the affective and motivational dimension of the pain experience in relation to anxiety and arousal.

Consistent with the concept of greater arousal and greater behavioral responses to an aversive pelvic visceral stimulus, we also found greater activation of the MCC in subjects with abuse, regardless of the presence of IBS symptoms (Figure 1). However, no significant difference in MCC activation by noxious visceral stimulus was seen between IBS patients and controls (Figure 2). The latter is in contrast to other reports showing greater activation of the dorsal cingulate in IBS patients. ${ }^{41,42}$ Possible explanations for this discrepancy include different patient populations (for example, greater proportion of subjects with abuse history in IBS and non-IBS groups, which diminish potential differences between the 2 groups, in predominant bowel habit or in gender) and previous exposure of subjects to the barostat procedure leading to habituation to the stimulus. ${ }^{43}$

In contrast to many somatic pain studies that have reported deactivation of the PCC associated with activation of the MCC during somatic pain stimuli, ${ }^{18-20}$ we found an increased activation of the PCC region immediately adjacent to the MCC. Wagner et $\mathrm{al}^{44}$ have reviewed information implicating a network consisting of the PCC and retrosplenial and medial parietal cortex to episodic memory retrieval. Of particular interest for the current findings is Wagner et al's hypothesis about the possible involvement of these brain regions in attention regulation to internal representations and memories. ${ }^{44}$ Consistent with this hypothesis, we have recently reported a reduction in the activation of this posterior attention network, including the PCC, following repeated rectal stimulation over a 12 -month period. ${ }^{43}$ Based on these findings, one may speculate that the observed increase in the activation of the PCC may be related to an enhanced attention to internal representation (memories of similar experiences) in patients with abuse history. Specific study paradigms should be applied to test this hypothesis.

Third, subjects with IBS and a history of abuse showed reduced activation of the SACC, a brain region involved in regulation of emotional arousal and indirectly, through its inhibitory effect on amygdala-related circuits, in pain inhibition. In contrast to the greater activation of the MCC/ PCC regions, the most rostral portion of the ACC, immediately above the genu of the corpus callosum showed less activation when subjects with IBS with abuse were compared with all others. These findings suggest that there is an interaction between abuse history and IBS in reducing 
activity in the sACC. In the psychiatric imaging literature, studies on brain responses in patients with posttraumatic stress disorders have been consistent in their findings of a reduced activation of the sACC, frequently associated with increased amygdala activity and with abnormal connectivity in the feedback interactions between these 2 brain regions involved in emotional regulation. ${ }^{14,45-48} \mathrm{~A}$ compromised ability to activate the sACC in response to emotional and pain stimuli may result in reduced inhibition of arousal and pain inhibition circuits. Different clinical sequelae of a similar trauma-induced impairment of arousal and pain regulation may include IBS and posttraumatic stress disorder in predisposed individuals.

Fourth, subjective pain ratings positively correlated with dorsal cingulate activation. Subjective pain reports during $50-\mathrm{mm} \mathrm{Hg}$ rectal distention across all 19 subjects showed a significant correlation with MCC and adjacent PCC activation, the same regions that showed greater activation in the abuse only and the IBS + abuse groups. These findings confirm previous reports of an association of dorsal cingulate activation and the subjective pain experience ${ }^{14,21,49}$ and are consistent with the concept that the dorsal cingulate mediates the affective and motivational dimension of an individual's pain response. These findings also provide a basis for further mechanistic studies relating psychosocial factors such as abuse history with increased pain reports via this region of the brain.

Activation in these dorsal cingulate subregions is associated with greater behavioral responses to an aversive visceral stimulus. Furthermore, the subjective pain ratings in our study also moderately correlated with the subjects' general level of anxiety, global psychologic distress, and, to some extent, depression. As discussed, the dorsal cingulate activation can be influenced by the amygdala and ascending noradrenergic projections from the locus coeruleus complex, which modulate the affective and motivational dimension of the pain experience in relation to anxiety and arousal.

Unlike many earlier previous descriptive brain-imaging studies, ${ }^{11-14}$ our current study is hypothesis driven based on previous data. ${ }^{15,32,42}$ The study results support the hypothesis that pain reports evoked by rectal distention is associated with specific predetermined regional brain activation and that a history of abuse enhances both pain reports and the activation at these brain regions. Furthermore, the increased activation of the MCC (an area associated with affective stimulus ratings and increased arousal) and the decreased activation in the sACC, an area associated with down-regulation of arousal and with endogenous pain inhibition, are in line with our a priori anticipated effects. Our study findings add to the previously reported observational data by suggesting a neurobiologic mechanism by which brain circuits concerned with visceral pain perception can be influenced by clinical and psychologic status.
In contrast to the ratings of pain intensity used in the current study, we have previously reported results from 2 psychophysiologic studies, which showed that rectal thresholds for both urgency and pain during rectal distention were not lower in IBS patients with abuse compared with IBS patients without a history of abuse. ${ }^{50,51}$ Differences in experimental paradigm (an ascending method of limits was used in the nonimaging studies), in the context of the experiment and in the stimulus rating tasks, may be responsible for the observed differences. However, one may speculate that the contrast between the effects of abuse history on pain threshold vs pain intensity ratings reflects a specific mechanism of central pain amplification.

Our study is limited by the relatively small number of subjects in each group. However, despite this limitation, type I error is unlikely for several reasons: (1) some of the study results are consistent with other observational studies, ${ }^{42}$ as well as (2) our a priori ROI hypotheses, and (3) ROI results were confirmed by whole-brain analyses. Because of the small number, we had insufficient power to determine whether the effect of abuse on pain reports persists when controlling for various psychologic comorbidities (eg, anxiety, global distress, and depression). However, the trends of higher depression in the abuse group and the correlation between reported pain and depressive symptoms may be meaningful given the small sample size and require further investigation in a larger sample. Another limitation is the fact that we did not study a non-IBS somatic control group. Even though this would have allowed us to determine whether the observed brain responses are specific to IBS or are observed with other functional pain syndromes, such an additional group, was beyond the scope of this study. Other limitations include the nonrandomized rectal distention protocol and the lack of control for menstrual cycle.

In conclusion, we demonstrated strong associations between visceral pain reporting and brain activation at predetermined brain regions that are involved in affective and motivational aspect of the human pain experience. We found a synergistic effect of IBS and abuse on subjective pain reporting and dorsal cingulate activation. Patients with both IBS and abuse not only reported significantly more pain during rectal distentions but also showed greater MCC and PCC activation and decreased sACC activation compared with all others. Our results point to enhancing effects of abuse history with IBS on an important region of the homeostatic afferent processing network in the brain and on pain reporting and suggest a possible mechanistic explanation to why patients with IBS and abuse report greater pain and poorer outcome. Further studies are needed to investigate whether these cortical activations are seen in other pain syndromes with abuse history, whether they may be responsive to medical and psychologic interventions, and 
whether this will be associated with better clinical outcome.

\section{References}

1. Drossman DA. Presidential address: gastrointestinal illness and the biopsychosocial model. Psychosom Med 1998;60: 258-267.

2. Drossman DA, Leserman J, Nachman G, et al. Sexual and physical abuse in women with functional or organic disorders. Ann Intern Med 1990;113:828-833.

3. Drossman DA, Li Z, Leserman J, et al. Health status by gastrointestinal diagnosis and abuse history. Gastroenterology 1996; 110:999-1007.

4. Talley NJ, Fett SL, Zinsmeister AR. Self-reported abuse and gastrointestinal disease in outpatients: association with irritable bowel-type symptoms. Am J Gastroenterol 1995;90:366371.

5. Walker EA, Gelfand AN, Gelfand MD, et al. Psychiatric diagnoses, sexual and physical victimization, and disability in patients with irritable bowel syndrome or inflammatory bowel disease. Psychol Med 1995;25:1259-1267.

6. Drossman DA. Irritable bowel syndrome and sexual/physical abuse history. Eur J Gastroenterol Hepatol 1997;9:327-330.

7. Drossman DA, Talley NJ, Olden KW, et al. Sexual and physical abuse and gastrointestinal illness: review and recommendations. Ann Intern Med 1995;123:782-794.

8. Drossman DA, Whitehead WE, Toner BB, et al. What determines severity among patients with painful functional bowel disorders? Am J Gastroenterol 2000;95:974-980.

9. Drossman DA. Do psychosocial factors define symptom severity and patient status? Am J Med 1999;107:S41-S50.

10. Leserman J, Drossman DA, Li Z, et al. Sexual and physical abuse history in gastroenterology practice: how types of abuse impact health status. Psychosom Med 1996:58:4-15.

11. Drossman DA. Brain imaging and its implications for studying centrally targeted treatments in IBS: a primer for gastroenterologists. Gut 2005;54:569-573.

12. Ringel $Y$. New directions in brain imaging research in functional gastrointestinal disorders. In a special issue on "Gl MotilityNew Directions." Dig Dis 2006;24:278-285.

13. Chang L. Brain responses to visceral and somatic stimuli in irritable bowel syndrome: a central nervous system disorder? Gastroenterol Clin North Am 2005;34:271-279.

14. Mayer EA, Naliboff BD, Craig AD. Neuroimaging of the brain-gut axis: from basic understanding to treatment of functional GI disorders. Gastroenterology 2006;131:1925-1942.

15. Drossman DA, Ringel Y, Vogt B, et al. Alterations of brain activity associated with resolution of emotional distress and pain in a case of severe IBS. Gastroenterology 2003;124:754-761.

16. Bremner JD. The relationship between cognitive and brain changes in posttraumatic stress disorder. Ann N Y Acad Sci 2006;1071:80-86.

17. Phillips ML, Gregory LJ, Cullen S, et al. The effect of negative emotional context on neural and behavioural responses to oesophageal stimulation. Brain 2003;126:669-684.

18. Peyron R, Laurent B, García-Larrea L. Functional imaging of brain responses to pain (a review and meta-analysis). Neurophysiologie Clinique 2000;30:263-288.

19. Derbyshire SWG. Metareview of functional imaging studies with noxious stimuli reveals reproducible patterns of central activation. J Pain 2001;2:655.

20. Apkarian AV, Bushnell MC, Treede RD, et al. Human brain mechanisms of pain perception and regulation in health and disease. Eur J Pain 2005;9:463-484.

21. Derbyshire SW. A systematic review of neuroimaging data during visceral stimulation. Am J Gastroenterol 2003;98:12-20.
22. Paus T. Primate anterior cingulate cortex: where motor control, drive and cognition interface. Nat Rev Neurosci 2001;2:417-424.

23. Derbyshire SW. Exploring the pain "neuromatrix." Curr Rev Pain 2000;4:467-477.

24. Craig AD. Interoception: the sense of the physiological condition of the body. Curr Opin Neurobiol 2003;13:500-505.

25. Maren S, Quirk GJ. Neuronal signalling of fear memory. Nat Rev Neurosci 2004;5:844-852.

26. Zubieta JK, Smith YR, Bueller JA, et al. Regional $\mu$ opioid receptor regulation of sensory and affective dimensions of pain. Science 2001;293:311-315.

27. Valet $\mathrm{M}$, Sprenger $\mathrm{T}$, Boecker $\mathrm{H}$, et al. Distraction modulates connectivity of the cingulo-frontal cortex and the midbrain during pain: an fMRI analysis. Pain 2004;109:399-408.

28. Leserman J, Drossman DA, Li Z. The reliability and validity of a sexual and physical abuse history questionnaire in female patients with gastrointestinal disorders. Behav Med 1995;21:141-150.

29. Leserman J, Li Z, Drossman DA, et al. Impact of sexual and physical abuse dimensions on health status: development of an abuse severity measure. Psychosom Med 1997;59:152160.

30. Leserman J, Li Z, Drossman DA, et al. Selected symptoms associated with sexual and physical abuse history among female patients with gastrointestinal disorders: the impact on subsequent health care visits. Psychol Med 1998;28:417-425.

31. Derogatis LR. SCL-90-R: Administration, scoring, and procedures manual II. For the R(evised) version. 1st ed. Towson: Clinical Psychometric Research, 1983.

32. Ringel $Y$, Drossman DA, Turkington TG, et al. Regional brain activation in response to rectal distention in patients with irritable bowel syndrome and the effect of a history of abuse. Dig Dis Sci 2003;48:1774-1781.

33. Azpiroz F, Bouin M, Camilleri M, et al. Mechanisms of hypersensitivity in IBS and functional disorders. Neurogastroenterol Motil 2007;19(Suppl 1):62-88.

34. Friston KJ, Holmes AP, Worsley KJ, et al. Statistical parametric maps in functional imaging: a general linear approach. Hum Brain Mapp 1995;2:189-210.

35. Friston KJ, Price $\mathrm{CJ}$, Fletcher $\mathrm{P}$, et al. The trouble with cognitive subtraction. Neuroimage 1996;4:97-104.

36. Drossman DA, Camilleri M, Mayer EA, et al. AGA technical review on irritable bowel syndrome. Gastroenterology 2002; 123:2108-2131.

37. Drossman DA. Sexual and physical abuse and gastrointestinal illness. Scand J Gastroenterol Suppl 1995;208:90-96.

38. Drossman DA, Creed FH, Olden KW, et al. Psychosocial aspects of the functional gastrointestinal disorders. In: Drossman DA, Corazziari E, Talley NJ, et al, eds. Rome II. The functional gastrointestinal disorders: diagnosis, pathophysiology and treatment. A multinational consensus. 2nd ed. McLean, VA: Degnon and Associates, 2000:157-245.

39. Santel S, Baving L, Krauel K, et al. Hunger and satiety in anorexia nervosa: fMRI during cognitive processing of food pictures. Brain Res 2006;1114:138-148.

40. Elman I, Sokoloff L, Adler CM, et al. The effects of pharmacological doses of 2-deoxyglucose on cerebral blood flow in healthy volunteers. Brain Res 1999;815:243-249.

41. Mertz H, Morgan V, Tanner G, et al. Regional cerebral activation in irritable bowel syndrome and control subjects with painful and nonpainful rectal distention. Gastroenterology 2000;118:842-848.

42. Naliboff BD, Derbyshire SWG, Munakata J, et al. Cerebral activation in irritable bowel syndrome patients and control subjects during rectosigmoid stimulation. Psychosom Med 2001;63:365-375.

43. Naliboff BD, Berman S, Suyenobu B, et al. Longitudinal change in perceptual and brain activation response to visceral stimuli in irritable bowel syndrome patients. Gastroenterology 2006; 131:352-365. 
44. Wagner AD, Shannon BJ, Kahn I, et al. Parietal lobe contributions to episodic memory retrieval. Trends Cogn Sci 2005;9:445-453.

45. Gilboa A, Shalev AY, Laor L, et al. Functional connectivity of the prefrontal cortex and the amygdala in posttraumatic stress disorder. Biol Psychiatry 2004;55:263-272.

46. Shin LM, Wright $\mathrm{Cl}$, Cannistraro PA, et al. A functional magnetic resonance imaging study of amygdala and medial prefrontal cortex responses to overtly presented fearful faces in posttraumatic stress disorder. Arch Gen Psychiatry 2005;62:273-281.

47. Rauch SL, Whalen PJ, Shin LM, et al. Exaggerated amygdala response to masked facial stimuli in posttraumatic stress disorder: a functional MRI study. Biol Psychiatry 2000;47: 769-776.

48. Armony JL, Corbo V, Clement $\mathrm{MH}$, et al. Amygdala response in patients with acute PTSD to masked and unmasked emotional facial expressions. Am J Psychiatry 2005;162:1961-1963.

49. Mayer EA, Berman S, Chang L, et al. Sex-based differences in gastrointestinal pain. Eur J Pain 2004;8:451-463.
50. Whitehead WE, Crowell MD, Davidoff AL, et al. Pain from rectal distention in women with irritable bowel syndrome: relationship to sexual abuse. Dig Dis Sci 1997;42:796-804.

51. Ringel Y, Whitehead WE, Toner BB, et al. Sexual and physical abuse are not associated with rectal hypersensitivity in patients with irritable bowel syndrome. Gut 2004;53:838-842.

Received May 7, 2007. Accepted October 18, 2007.

Address requests for reprints to: Yehuda Ringel, MD, University of North Carolina at Chapel Hill, 1140 Bioinformatics BIdg, 130 Mason Farm Road, Chapel Hill, North Carolina 27599. e-mail: ringel@ med.unc.edu; fax: (919) 843-0800.

Supported by K23 DK075621 (to Y.R.), DK48351, R01 MH46959 (to D.D.), R24 DK067674 (to W.E.W. and D.D.), R24 AT002681 (to E.A.M.), P50 DK64539 (to E.A.M.), and R01 DK48351 (to E.A.M.). Conflicts of interest: No conflicts of interest exist. 